https://doi.org/10.46344/JBINO.2020.v09i06.41

\title{
HOME HYGIENE PRODUCT FOR EXFOLIATING FEET-Review Article
}

----A warm alkaline hypertonic solution of hydrogen peroxide containing oxygen gas at excessive pressure urgently whitens and exfoliates thickened skin and nail plate.

\section{1,2A.L.Urakov}

'Department of Modeling and Synthesis of Technological Structures Udmurt Federal Research Center of the Ural branch Russian Academy of Sciences; 2Department of General and Clinical Pharmacology of the Izhevsk State Medical Academy of the Ministry of health Russian Federation, Izhevsk, Russia - 426034

E-mail: urakoval@live.ru

\section{ABSTRACT}

To exfoliate excess keratin in the skin and nail plates of the foot, folk remedies have long been used, among which warm baths, alkaline Soaps, shampoos and pumice are still in the lead. But these products do not provide high speed and efficiency of exfoliation. In this regard, in 2020, Russia developed and patented a warm alkaline hypertonic solution of hydrogen peroxide, additionally enriched with oxygen gas, which exceeds the effectiveness of all previously known exfoliators. This solution is a hygiene product for the feet and can be used at home using warm baths. The solution contains known and safe ingredients, namely-3.0-5.0\% potassium hydroxide, $0.5-20 \%$ hydrogen peroxide, water and oxygen gas under an excess pressure of 0.2 ATM at $+8^{\circ} \mathrm{C}$. Before application, the solution is heated to a temperature of $+38-+42{ }^{\circ} \mathrm{C}$. The solution has a $\mathrm{pH}$ of 13.0-14.0, osmotic activity of 350-560 mosmol// of water. Immersion of the foot in this solution for 5 - 15 minutes provides rapid softening of the keratinized layer of the epidermis in the area of dry calluses, softening of the excess deformed nail plate in case of fungal nail damage, discoloration of the skin in the area of bruising, restoring elasticity, smoothness, skin color, shape and color of the deformed nail plate and improving the aesthetic result without tanning, coloring, local irritating and cauterizing effects.

Key words: drugs development, exfoliation, bleaching, deodorization, hyperkeratosis, fungal disease, hygiene product. 


\section{Introduction}

The modern arsenal of ready-made hygiene and cosmetic products is quite large. However, pharmacies do not have fast-acting hygiene and cosmetic products for peeling with hyperkeratosis of the feet [1]. Unfortunately, official medicine do not yet offer effective hygiene products that provide emergency bleaching, exfoliation of the skin of the feet with hyperkeratosis and nail plates with fungal lesions and/or with bruises [2]. Under these conditions, the victims traditionally use folk remedies, including pumice, hot water baths, alkaline shampoos and soaps to treat nails and skin areas that are thickened by hyperkeratosis or have changed their color due to bruises, hematomas or fungal diseases [3]. However, these tools do not have a high speed of action. In this regard, the aesthetic condition of the feet, worsened by hyperkeratosis, fungal disease, bruising and hematoma, can not be improved in a few minutes. Therefore, victims are forced to accept the deterioration of the aesthetic condition of the feet for a long period of time. However, few of them seek medical help, although they are forced to hide the affected feet from the eyes of people around them. This leads to the progression of the pathology and is often complicated by the appearance of cracks, itching and pain in the feet, and also creates discomfort for people when walking.

The aim of the study was to find hygienic and cosmetic products that provide urgent softening and reducing the thickness of the skin and nail plate by exfoliating the layer of dry and hard epidermis and nail with excess deformation. At the same time, it was expected to find means that can restore the natural color, elasticity, smoothness and shape of the skin and nail area in hyperkeratosis, fungal disease, bruising, hematoma under the skin and/or under the nail plate using food, hygiene, cosmetic products and medicines.

One of the first hygiene products developed for the treatment of foot hyperkeratosis at home was a dry mixture with a particle size of 0.1-2.5 mm, including $\quad 0.1-44.9 \% \quad$ potassium permanganate and natural salt - the rest. At the same time, it was recommended to use sea and/or rock salt, and/or table salt, and/or salt of salt lakes as natural salt. The product may additionally contain food coloring agents, various essential oils and / or extracts of medicinal plants in an effective amount [4]. However, this product does not provide an emergency washing and exfoliating effect for hyperkeratosis of the feet. The fact is that the product is not a ready-made drug solution. In addition, about half of the dry mass of the known product is potassium permanganate. Therefore, the product stains the skin of the hands and feet, as well as clothing and dishes of the consumer in a crimsonbrown color and poses a risk of ignition of oxidized substances during local interaction. Due to the uncertain composition and uncertain concentrations of ingredients, the known agent may not be sufficiently active in some cases. Therefore, some people refuse to re-use a known remedy for treating feet. 
Also, for the treatment of hyperkeratosis of the feet at home, a liquid remedy was proposed consisting of 1 liter of hot water, a tablespoon of soap shavings and 2-3 teaspoons of soda, or 1.5 liters of hot water and 4 large spoons of hydrogen peroxide [5]. But this remedy also does not have an exact recipe, so it does not have a reliable therapeutic effect.

In addition to these remedies for the treatment of various skin diseases of the feet at home, it was proposed to use a therapeutic composition that contains up to 5 wt.\% organosilane Quaternary compounds and up to $20 \%$ hydrogen peroxide [6]. However, this product also does not have an exact formulation and does not contain alkali, so it does not provide a stable pharmacological effect and emergency softening and exfoliation of the hyperkeratosis area in the skin and urgent discoloration of the skin and nails with bruises and bruises.

Regardless of these hygiene products, a fast keratin solvent was proposed for industrial use, which is an aqueous solution of $10-30 \%$ potassium hydroxide [7]. This solution of caustic potassium has a very high alkalinity and is therefore able to dissolve keratin. But this remedy does not have an exact formulation, does not contain hydrogen peroxide and a local anesthetic that easily penetrates the skin, so it does not have high effectiveness and safety when applied topically. In particular, it has a pronounced local irritant effect, has an ulcerogenic effect on the skin and can cause ulcers of the skin of the foot.

In 2020, Russia developed and patented a product for urgent peeling for hyperkeratosis of the feet, which provides urgent exfoliation and bleaching of the skin and nail plates of the feet for hyperkeratosis, fungal disease and bruises. It was shown that this effect can be achieved at the expense of local use warm alkaline hypertonic solution of hydrogen peroxide, which additionally contains oxygen gas under high pressure [8]

The essence of this tool is that the components are contained in it at the following ratio (wt. \%):

Potassium hydroxide $\quad-3,0-5,0$

Hydrogen peroxide - 0,5-20,0

Oxygen - until overpressure is created 0,2 ATM at $+8^{\circ} \mathrm{C}$.

Water - the rest at a $\mathrm{pH}$ of 13.0 - 14.0, osmotic activity of $350-560 \mathrm{mosmol} / \mathrm{l}$ of water and local temperature $+38-+42^{\circ}$ C.

It has been shown that this hygiene product loosens and dissolves hardened keratin in the skin and in the nail plate, has a deodorizing, discoloring and washing effect, and does not have a coloring, local irritant, ulcerogenic and cauterizing effect.

At the same time, it was found that the mechanism of local action of this tool is as follows. It was shown that the product is an aqueous alkaline solution with oxidative, saponifying, loosening, dissolving, bleaching, washing and pronounced penetrating power, providing hyperthermic melting, alkaline dissolution and oxidative bleaching of keratin, blood clots and pus, sterilizing effect on the treated surface, physical and chemical and interstitial cavitation loosening, flotation removal of organic masses from the surface and in the thickness of the epidermis while washing 
and bleaching the surface layer of the skin.

In particular, the use of the solution at a temperature of $+38-+42^{\circ} \mathrm{C}$ provides an increase in the efficiency and safety of the foot skin cleaning process due to local hyperthermia, which accelerates and enhances cleaning due to the temperature dependence of the rate of chemical, physico-chemical and biochemical reactions according to the Arrhenius law. The proposed temperature range is physiological and optimal, since local hyperthermia implies a temperature exceeding normal values, that is, above $+37^{\circ} \mathrm{C}$, and an increase in temperature above $+42^{\circ} \mathrm{C}$ becomes dangerous for living cells of the human body, since such local hyperthermia can cause a temperature burn of tissues in the area of cracks and wounds of the skin.

The use of a solution of potassium hydroxide at a concentration of 3-5\% provides alkalinity in the $\mathrm{pH}$ range of $13.0-$ 14.0, osmotic activity in the range of 340 $550 \mathrm{mosmol} / \mathrm{l}$ of water and the ability to quickly dissolve keratin in combination with $0.5-20.0 \%$ hydrogen peroxide with an excess oxygen gas content. At the same time, hydrogen peroxide and oxygen gas are environmentally safe means for the environment.

The specified range of caustic potassium concentration values provides pronounced alkaline and moderate hypertonic activity, which is sufficient for disinfection of the foot, but excludes ulcerogenic effect on its skin with a single short-term exposure (lasting up to 15 minutes). At the same time, this concentration of caustic potassium increases the environmental safety of the product. The fact is that the content of caustic potassium in a concentration of less than $3 \%$ does not provide effective hypertonic, that is, hyperosmotic activity of the solution. On the other hand, the content of caustic potassium in the solution at a concentration of more than $5 \%$ gives the solution an excessively high hyperosmotic activity, which can cause tissue burns due to dehydration damage to cells in the epithelial layer and/or granulation tissue cells. In addition, the content of potassium hydroxide more than $5 \%$, turns the solution into an excessively aggressive alkaline agent, which has a detrimental effect on the microflora and plants in the environment when pouring the used product on the ground.

It is shown that the use of $0.5-20 \%$ hydrogen peroxide in the solution gives it the necessary oxidative, cavitation and bleaching activity while maintaining the safety of the solution for human skin. The fact is that solutions containing hydrogen peroxide in a concentration of up to $20 \%$ do not have a local irritating effect [9].

It has been demonstrated that when this solution is poured from a closed container into a basin or bath, oxygen gas bubbles immediately begin to appear in the solution, and the "cold boiling"process begins. Then, when the solution comes into contact with a tissue containing the enzyme catalase, oxygen gas bubbles additionally begin to form in the solution, which appears from hydrogen peroxide as a result of the catalase reaction. This usually occurs when there are traces of blood and / or pus on the surface of the skin of the feet.

It has been shown that immediately after the foot is immersed in this solution, it immediately begins to dissolve and 
remove liquid, viscous and dense biological masses from the entire surface of the skin, from the surface of the nail plates and even from under them and from cracks, and simultaneously disinfects and deodorizes the surface. Therefore, the solution whitens the skin in the area of bruises and eliminates the bad smell coming from the feet, especially with fungal lesions.

It is important to note that this solution contains oxygen gas under high pressure, which provides cavitation due to the formation of oxygen gas bubbles in the entire volume of the liquid, but excludes excessively rapid boiling and splashing out of the "working" chamber (basin or bath). Hydrogen peroxide provides interstitial release of molecular oxygen, the formation of gas bubbles (cavitation) in the entire volume, as well as in the thickness of molten keratin, while simultaneously destroying the "monolithic" structure of the biological mass due to interstitial cold boiling with the formation of oxygen gas bubbles. In this case, the bubbles adhere to the loosened and crushed particles of these masses, these particles are separated from the surface of the living skin layer by floating bubbles (flotation) and mixing the solution with the liquefied and crushed particles of biological masses. In addition, oxygen oxidizes organic pigments and discolors them. All this increases the effectiveness of removing excess keratin in the skin of the feet in the area of hyperkeratosis, as well as in the nail plate deformed by the fungus, and ensures the safety of the drug for healthy skin areas. Under these conditions, molecular oxygen is rationally used for loosening a thick, infected and rotting biological mass under the influence of microorganisms, consisting of keratin and other organic residues (sweat, pus, blood), since the agent provides a cold "boiling" inside this mass. This ability of an alkaline aqueous solution of hydrogen peroxide to dissolve and bleach thick pus, dried blood, and rotting food residues (organic substances) with local interaction was described earlier $[10,11,12]$.

This hygienic drug used as follows. The drug is heated to the selected level of hyperthermia and immediately poured into a container (basin or bath), in which the foot (or both feet) is immediately immersed and left in it for 5 to 15 minutes. After completing the procedure, the foot is washed with water and wiped with a towel.

\section{Conclusion}

Thus, for peeling with hyperkeratosis of the legs, a warm alkaline hypertonic solution of hydrogen peroxide, additionally enriched with oxygen gas, was developed. This new medicine is a hygienic foot product and can be used at home with warm baths for urgent peeling. The new tool was developed and first patented in Russia in 2020. It contains known and safe ingredients, namely-3.0 - 5.0\% potassium hydroxide, $0.5-20 \%$ hydrogen peroxide, water and oxygen gas under an excess pressure of 0.2 ATM at $+8^{\circ} \mathrm{C}$. This solution has a $\mathrm{pH}$ of 13.0-14.0, osmotic activity of 350-560 mosmol/l of water. Immersion of the foot in this solution, heated to a temperature of $+38-+42{ }^{\circ} \mathrm{C}$, provides rapid softening of the keratinized layer of the epidermis in the area of dry calluses, softening of excess deformed nail plate with fungal nail damage, discoloration of the skin in the area of bruising, restoring elasticity, 
smoothness, skin color, shape and color of the deformed nail plate and improving the aesthetic result without tanning, coloring, local irritating and cauterizing effects.

Conflict of interest: None declared.

\section{Reference}

1. Garcia CA, Soler FC. The effect of plantar hyperkeratosis debridement on self-perception of pain levels in older people. International Journal of Gerontology. 2018; 12(4): 314318.

\section{https://doi.org/10.1016/j.ijge.2018.05.002.}

\section{Bianch J, Page B, Robertson}

S. Your Dermatology Pocket Guide: Common skin conditions explained. NHS. Education for Scotland. 2011.

3. Tarun J, Susan J, Suria J, Susan VJ, Criton S. Evaluation of $\mathrm{pH}$ of bathing soaps and shampoos for skin and hair care. Indian J Dermatol. 2014:59(5):442-444. doi:10.4103/00195154.139861.

4. Kozlova OA. Agent for preparing solution for baths, for foot and hand baths, oral care, for disinfecting water. RU Patent 2593794. 27.09.2013.

5. Foot baths: effective recipes. https://beautydream.ru/ped/vannochki.h tml.

6. Dzherom JKh, Govard OG. Therapeutic composition for treating skin diseases, containing organosilane quaternary compounds and hydrogen peroxide. RU Patent 2380102. 27.01.2010.

7. Vorobiev AA. Medical and Sanitary Microbiology. Moscow: publishing center «Academy», 2003. https://www.academiamoscow.ru/ftp_share/_books/fragments/f ragment_18870.pdf.
8. Urakov AL, Alies MYu, Samilina IA e.al. Peeling agent for foot hyperkeratosis. RU Patent 2730451. 24.08.2020.

9. Molenda MA, Sroa N, Campbell SM, Bechtel MA, MD, Opremcak EM. Peroxide as a novel treatment for ecchymoses. J Clin Aesthet Dermatol. 2010; 3(11): 36-38.

10. Urakov A., Urakova N., Reshetnikov A. Oxygen alkaline dental's cleaners from tooth plaque, food debris, stains of blood and pus: A narrative review of the history of inventions. Journal of International Society of Preventive \& Community Dentistry. 2019, V. 9. N 5. P. 427-433.

11. Urakov AL. Medications and bruises: past, present, and future. Reviews of clinical pharmacology and drug therapy. 2020; 18(2):161-170. https://doi.org/10.7816/RCF182161-170.

12. Urakov $A L$, Urakova NA. Hydrogen peroxide discolors blood in the cavity of the hematoma under the nail and in the skin over the bruise. Regional Blood Circulation and Microcirculation. 2020;19(2):67-74. (In Russ.) https://doi.org/10.24884/1682-6655-202019-2-67-74. 